\title{
Periodontal Disease in Five and Six Year Old Children
}

\author{
A. John Spencer, ${ }^{*}$ David Beighton, $\dagger$ and Thomas J. Higgins $\ddagger$
}

Accepted for publication 28 March 1982

\begin{abstract}
THE PURPOSE of this study was to determine the prevalence and severity of gingivitis in a sample of primary school children. One hundred and twenty eight children with an average age of 6 years 0 months ( $S D \pm 4$ months) were examined for oral hygiene status using the Plaque Index (Pl.I.) and the Calculus Index (C.I.). The periodontal status was assessed using the Gingival Index (G.I.) and recording gingival sulcus depths about selected teeth. The significance of factors associated with the G.I. was determined using multiple classification analysis. The incorporated factors were Pl.I., age and sex. The scores for Pl.I., and G.I., and Pl.I. and sulcus depth were significantly correlated. The majority of children had a maximum Pl.I. score of 2, a maximum C.I. score of 0 , a maximum G.I. score of 1 , and maximum sulcus depth of $2 \mathrm{~mm}$. The regressands P1.I. and age used in the multiple classification analysis explained only $28 \%$ of the variance in the G.I. data. Also, the analysis did not indicate the children's sex as a significant source of explanation for the variance of the G.I.
\end{abstract}

Periodontal diseases are present in almost all persons with natural teeth. From an early age, gingivitis increases in its severity to peak at onset of puberty. Thereafter, as the severity of gingivitis decreases, chronic periodontitis measured by loss of attachment becomes dominant and continues to increase in severity.

The control of gingivitis in young children by its early detection, prevention and treatment may be a realistic approach to the reduction of the prevalence and severity of chronic periodontitis in adulthood. ${ }^{1,2}$ The assumption that periodontitis has its aetiology as gingivitis in children awaits a complete understanding of the natural history of these periodontal diseases. ${ }^{3}$ However, even if the inception of chronic periodontitis is found to occur after the childhood years, common factors may be associated with gingivitis in children and chronic periodontitis in adults. These factors may be susceptible to modification.

The purpose of the present study was to determine the prevalence and severity of gingivitis in a sample of primary school children, and discuss the factors which may explain any variation in gingivitis in children.

* Australian National Health and Medical Research Council Fellowship in Applied Health Sciences, Department of Conservative Dentistry, Faculty of Dental Science, University of Melbourne, 711 Elizabeth St, Melbourne, Victoria, 3000 Australia.

$\dagger$ Dental Research Unit, Royal College of Surgeons of England, Downe, Kent, United Kingdom.

₹isiting Assistant Professor, The University of Michigan School of Dentistry, Department of Periodontics, Ann Arbor, MI 48109 U.S.A.

\section{MATERIALS AND METHODS}

\section{Survey Population}

A sample of 128 primary school children (71 males and 57 females) with an average age of 6 years 0 months (SD \pm 4 months) was obtained from the primary grade classes of five government schools in and around Melbourne, Australia. Three of the schools were located in a rural area outside Melbourne and two were metropolitan schools giving a sample of 60 rural and 68 urban children.

\section{The Dental Examination}

Standardized inspection type examinations were carried out in the schools by one of the authors (A.J.S.). Oral hygiene was assessed using the Plaque Index (Pl.I.) and Calculus Index (C.I.). ${ }^{4}$ Periodontal status was assessed using the Gingival Index (G.I.) ${ }^{4}$ and periodontal sulcus depth was measured using a William's No. $14 \mathrm{~W}$ periodontal probe. No attempt was made to determine loss of attachment. The Pl.I., C.I., G.I., and sulcus depths were assessed on the following primary teeth: upper right second molar, upper right lateral incisor, upper left first molar, lower left second molar, lower left lateral incisor, and lower right first molar. The selection of these primary teeth was based on a partial mouth recording system suggested for the permanent dentition, ${ }^{5}$ which aimed at obtaining measurements from all segments of the mouth. If a selected primary tooth had exfoliated, 
the successor was observed, provided it had erupted into occlusion. Missing primary second molar teeth were substituted for by the adjacent first permanent molar if the latter had erupted. For the Pl.I., G.I., and sulcus depth, four surfaces (mesial, buccal, distal and lingual) were observed per selected tooth. Only two surfaces (buccal and lingual) were observed per selected tooth for the C.I.

Analysis

All data were recorded directly onto a field record form in numerical code, transferred to 80 column computer data cards and analyzed using the University of Melbourne's Control Data Cyber computer and program procedures from the Statistical Package for the Social Sciences. ${ }^{6}$ One way analysis of variance or the appropriate Student's $t$ test were used to compare mean values of the Pl.I., C.I., G.I. and sulcus depths for groups formed by whether substitution of permanent teeth had occurred or not, and by the children's sex. Correlation coefficients were calculated using Spearman's correlation coefficient. The significance of factors associated with the G.I. were

Table 1

Mean and Standard Deviation of P1.I., G.I. and Sulcus Depth (mm) For 19 Children Aged 5 and 6 Years Old With and Without Substitution of Permanent Teeth for Missing Primary Teeth

\begin{tabular}{lccc}
\hline & $\begin{array}{c}\text { With substitu- } \\
\text { tion }\end{array}$ & $\begin{array}{c}\text { Without substi- } \\
\text { tution }\end{array}$ & $r$ \\
\hline Pl.I. & $0.68 \pm 0.24$ & $0.66 \pm 0.24$ & $0.88^{*}$ \\
G.I. & $0.71 \pm 0.25$ & $0.66 \pm 0.29$ & $0.87 \dagger$ \\
Sulcus depth & $0.90 \pm 0.20$ & $0.87 \pm 0.28$ & $0.68 \dagger$ \\
\hline
\end{tabular}

$* P<0.001$.

$\dagger P<0.01$. determined using multiple classification analysis. Factors incorporated in the analysis included Pl.I., age and sex.

\section{RESULTS}

The effects of substituting a permanent tooth for a missing primary tooth on the average scores for the Pl.I., G.I. and sulcus depth were investigated before proceeding with the analysis. The mean Pl.I., G.I. and sulcus depth were determined both with and without substituted permanent teeth for the 19 children where some substitution had taken place. These data are presented in Table 1. There was no significant difference between the means of the two sets of scores for each variable and the pairs of scores were significantly correlated in all instances. The C.I. was not compared with and without substitution because only $8 \%$ of all children had evidence of any calculus.

The frequency distributions of children by their maximum score among all observations of Pl.I., C.I., G.I. and sulcus depth are illustrated in Figure 1. The majority of children had a maximum Pl.I. score of 2 , a maximum C.I. score of 0 , a maximum G.I. score of 1 , and a maximum sulcus depth of $2 \mathrm{~mm}$. The mean Pl.I., G.I. and sulcus depth of the whole sample of 128 children and for both sexes separately are presented in Table 2 . There were no significant differences between male and female scores for any of the oral hygiene or periodontal status variables. As the prevalance of calculus was so low a mean score was not presented.

The scores for Pl.I. and G.I., and Pl.I. and sulcus depth were significantly correlated. However, there was no significant association between sulcus depth and G.I. These data are presented in Table 3.

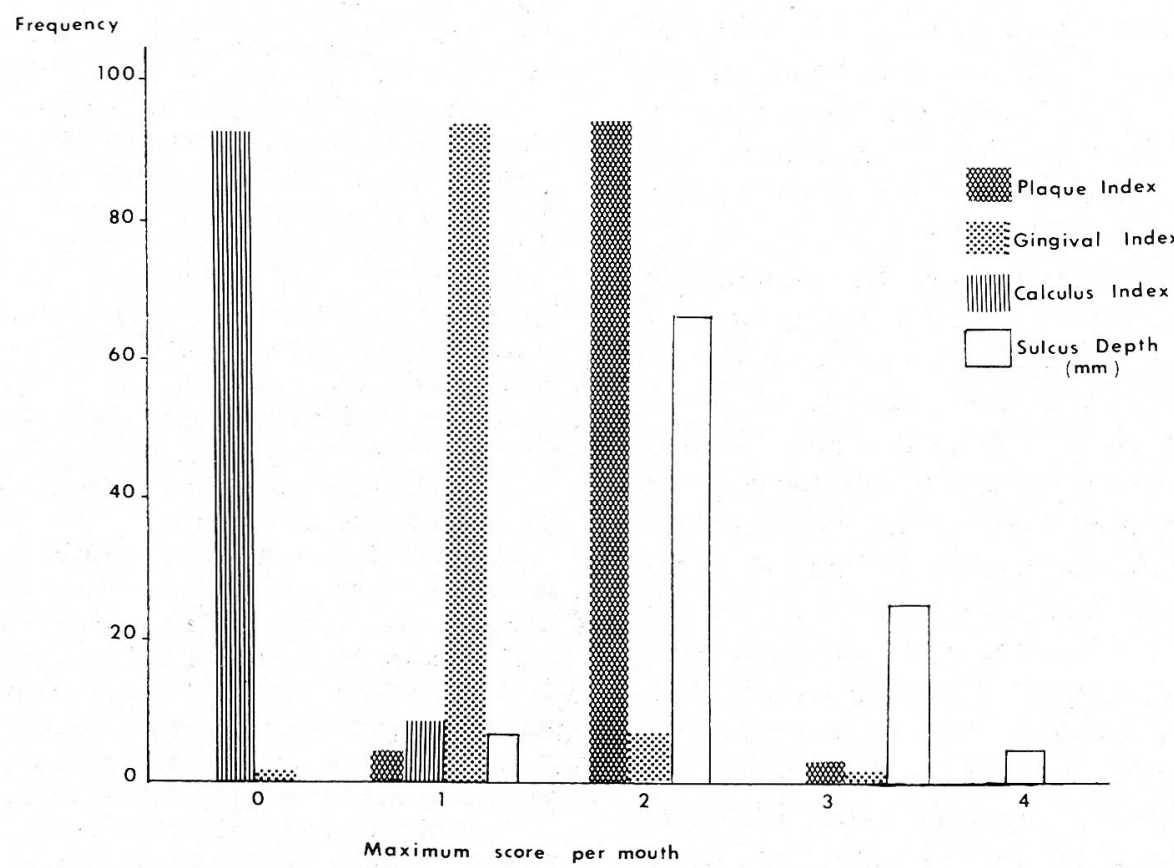

Figure 1. Frequency distribution of children by their maximum scores for all observations of PI.I. C.I., G.I. and sulcus depth. 
Table 2

Mean and Standard Deviation of P1.I., G.I. and Sulcus Depth of 128 Children Aged 5 and 6 Years Old

\begin{tabular}{lccc}
\hline & All $N=128$ & Males $N=71$ & $\begin{array}{c}\text { Females } N= \\
57\end{array}$ \\
\hline Pl.I. & $1.09 \pm 0.39$ & $1.04 \pm 0.44$ & $1.15 \pm 0.32$ \\
G.I. & $0.60 \pm 0.24$ & $0.58 \pm 0.24$ & $0.64 \pm 0.23$ \\
Sulcus depth & $0.90 \pm 0.28$ & $0.92 \pm 0.30$ & $0.90 \pm 0.25$ \\
\hline
\end{tabular}

Table 3

Correlation Matrix for P1.I., G.I. and Sulcus Depth in 128 Children Aged 5 and 6 Years Old

\begin{tabular}{llll} 
& P1.I. & G.I. & $\begin{array}{c}\text { Sulcus } \\
\text { depth }\end{array}$ \\
\hline P1.I. & 1.00 & & \\
G.I. & $0.44^{*}$ & 1.00 & \\
Sulcus depth & $0.47^{*}$ & 0.16 & 1.00 \\
\hline
\end{tabular}

$* P<0.001$.

The explanatory variables or regressands used in the multiple classification analysis were Pl.I., age and sex. Both Pl.I. and age were divided into three categories based on the distribution of data for the regressand, while sex was entered as a two category regressand. Only Pl.I. and age emerged as significant sources of explanation of the variance of the dependent variable or regressor, G.I. The $\beta$ coefficients for Pl.I. and age were 0.45 $(P<0.001)$ and $0.21(P<0.01)$ respectively. The $\mathrm{R}^{2}$ value was 0.28 indicating that the regressands explained $28 \%$ of the variance in the G.I. data. The analysis failed to indicate the children's sex as a significant source of explanation for the variance of the G.I. scores.

\section{DISCUSSION}

Although the eruption of permanent teeth into the oral cavity has been associated with an increased inflammatory response in the gingival tissue, ${ }^{7}$ the present study failed to demonstrate any significant difference in G.I., Pl.I., or sulcus depth scores when permanent molars were substituted for missing primary molars.

The high prevalence of a mild gingivitis in the sample of 5 and 6 years olds agrees with the observation of other investigations. ${ }^{8-10}$ The maximum G.I. score on each child characterized the periodontal status as one of nearly universal presence of signs of gingivitis but with little bleeding being provoked by pressing against the gingivae with the side of a periodontal probe. The maximum G.I. scores were also consistent with the observed sulcus depths of most children being 2 or $3 \mathrm{~mm}$ in depth. The low mean scores for Pl.I., G.I., and sulcus depth indicated that many children had segments of their mouths with considerably better oral hygiene and periodontal status than indicated by the maximum score distributions.

The simple correlation between Pl.I. and G.I. scores for this study was low, although statistically significant. The correlation between Pl.I. and G.I. in an adolescent sample has been reported as $0.6^{11}$ and as high as 0.9 in adults. ${ }^{12}$ With a low simple correlation between Pl.I. and G.I. and a narrow age range in the sample, the finding that Pl.I. and age combined explained only $28 \%$ of the variation in G.I. was not unexpected.

The weak correlation between G.I. and Pl.I. in the 5 and 6 year old children in this study suggests that even reasonably poor oral hygiene in such children may not result in the expected severity of gingivitis. The explanation for the weak correlation between the G.I. and Pl.I. may be associated with the anatomy of primary molars at the gingival third as compared to permanent premolar and molar teeth. ${ }^{13}$ Variations may also exist between the microbial flora in the gingival crevice of children and adults and in the intensity of the host reactions to commensal flora. ${ }^{14-17}$ The prevalence of Spirochaetes and Bacteroides melaninogenicus is lower in children aged 3 to 7 years than in adults. ${ }^{18}$

In conclusion, this study indicated that the prevalence of periodontal disease in a sample of 5 and 6 year old children was high, but severity was low. The relation between plaque accumulation and the gingival inflammation was found to be weaker than in adults. Reductions in plaque accumulation may not be rewarded by reductions in gingival inflammation associated with the primary dentition. Programs of prevention and treatment of periodontal disease in children may be justified best by the introduction of desired habits.

\section{REFERENCES}

1. McCall, J. O.: Gingival and periodontal disease in children. $J$ Periodontol 9: 7, 1938.

2. Muhlemann, H. R., and Son, S.: Gingival sulcus bleeding - a leading symptom in initial gingivitis. Helv Odont Acta 15: 107, 1971.

3. Sheiham, A.: Screening for periodontal disease. J Clinol Periodontol 5: 237, 1978.

4. Löe, H.: The Gingival Index, the Plaque Index and the Retention Index systems. J Periodontol 38: 610, 1967.

5. Ramfjord, S. P.: Indices for prevalance and incidence of periodontal disease. J Periodontol 30: 51, 1959.

6. Nie, N. J., Hull, C. A., Jenkins, J. G., Steinbrenner, K., and Bent, D. H.: Statistical Package for the Social Sciences. ed 2, New York, McGraw-Hill, 1975.

7. Kelly, J. E., and Sanchez, M. J.: Periodontal disease and oral hygiene among children. Vital Health Statistics. Series 11-No. 117. DHEW Pub. No. (HSM) 72-1060. Washington, U.S. Government Printing Office, 1972.

8. Holm, A. K.: Oral health in five year old Swedish children. Community Dent Oral Epidemiol 3: 184, 1975.

9. Köhler, L. and Holst, K.: Dental health of four year old children. Acta Paediat Scand 62: 269, 1973.

10. Lindhe, J. and Axelsson, P.: The effect of controlled oral hygiene and topical fluoride application on caries and gingivitis in Swedish school children. Community Dent. Oral Epidemiol. 1: 9, 1973.

11. Sanchez, M. J.: Periodontal disease among youths 12-17 years. Vital Health Statistics Series 11-No. 141, DHEW Pub. No. HRA 741623. Washington, U.S. Government Printing Office, 1974.

12. Russell, A. L.: International nutritional surveys. A summary of preliminary dental findings. $J$ Dent Res 42: 233, 1963.

13. Parfitt, G. J.: Periodontal diseases in children. pp 286-308. Finn, S. B. (ed), Clinical Pedodontics, ed 4, pp 286-318. Philadelphia, W. B. Saunders Company, 1973. 


\section{Spencer, Beighton, Higgins}

14. Matsson, L.: Development of gingivitis in some school children and young adults. Comparative experimental study. J Clin Periodontol 5: 24,1978 .

15. Smith, L. B., Golub, L. B., and Duperon, D. F.: An evaluation of crevicular fluid and gingival tissue in children. J Dent Child 41: 128, 1974.

16. Biswas, S., Duperon, D. F., and Chebib, F. S.: Study of periodontal disease in children and adolescence. I. Effects of age, sex and gingival inflammation on crevice fluid volume, pocket depth, $\mathrm{pH}$, supragingival plaque and crevice collagenase activity and urea. $J$ Periodontol Res 12: 205, 1977.
17. Longhurst, P., Johnson, N. W., and Hopps, R. M.: Differences in lymphocyte and plasma cell densities in inflamed gingiva from adults and young children. $J$ Periodontol 48: 705, 1977.

18. DeAraujo, W. C., and McDonald, J. B.: The Gingival crevice microbiota of preschool children. J Periodont 35: 285, 1954.

Send reprint requests to: Dr. A. John Spencer, Department of Conservative Dentistry, University of Melbourne, 711 Elizabeth St, Melbourne, Victoria 3000 Australia.

\section{In Memoriam}

\section{Frederik L. Hansen, D.D.S. 1901-1981}

Dr. Frederik L. Hansen, a practicing dentist in Chicago for several decades, was born in Copenhagen, Denmark and educated in Chicago, where he graduated from Northwestern University Dental School in 1922. He was especially interested in the role of occlusion in the treatment of periodontal diseases, and gave many lectures on this subject before various dental organizations.

Survivors include his wife, Mrs. A. Hansen.

\section{J. Milton Orlando, D.D.S., M.S. 1939-1981}

Dr. J. Milton Orlando, a periodontist from San Antonio, Texas, died October 21, 1981. He received his D.D.S. degree from the University of Texas and his graduate training in periodontology at the University of Missouri (M.S. 1970). He was elected to membership in the Academy in 1972.

No further details are known.

\section{Ralph E. Stoker, D.D.S. 1900-1981}

Dr. Ralph E. Stoker of Huntington Park, California, was born in Piqua, Ohio, in 1900 and received his D.D.S. from the University of Southern California in 1925. In later years he limited his practice to periodontics and taught at the University of Southern California part-time in 1950 and 1951 . He became a member of the Academy of Periodontology in 1948. Dr. Stoker died suddenly in the fall of 1981.

Survivors include his wife, Florence, and son, Donald. 